\title{
ANÁLISIS TURÍSTICO BASADO EN LAS FORTALEZAS Y DEBILIDADES INTRÍNSECAS DEL TERRITORIO: CASO ZARUMA, ECUADOR
}

\author{
TOURIST ANALYSIS BASED ON STRENGTHS AND \\ INTRINSIC WEAKNESSES OF THE TERRITORY: \\ ZARUMA CASE, ECUADOR
}

Luis Miguel Sánchez Cuenca

Universidad Técnica de Machala, Ecuador

Abraham Isaías Parra Prado

Universidad Técnica de Machala, Ecuador

Melissa Paulina Calle Iñiguez

Universidad Técnica de Machala, Ecuador

Aceptado: 14 de enero de 2020

\section{RESUMEN}

La potencialidad turística se basa en los aspectos intrínsecos que posee un recurso o destino del territorio en función de la actividad, para desarrollarlas por medio de una correcta gestión. En este contexto, el presente estudio se enfoca en cinco parroquias del cantón Zaruma, con el objetivo de determinar las principales fortalezas o debilidades que fomentan u obstaculizan el turismo en el sector. El análisis se basa en los lineamientos de la Guía metodología para la jerarquización de atractivos y generación de espacios turísticos del Ecuador. Se empleó la primera etapa con sus dos fases iniciales, y para facilitar el análisis de los criterios, se aplicó un porcentaje de ponderación a los criterios de evaluación para realizar gráficos estadísticos de los atractivos, lo que permitió identificar que el potencial turístico radica en la modalidad turística cultural-patrimonial y que sus atractivos complementarios, a pesar de tener muchas debilidades, pueden ser desarrollados con una gestión adecuada.

Palabras clave: potencialidad turística, planificación, criterios de evaluación, diagnostico turístico, desarrollo sustentable, turismo.

\section{ABSTRACT}

This study focuses on five parishes in the Zaruma canton, with the aim of determining the main strengths or weaknesses that promote or hinder tourism in the sector. The analysis is based on the guidelines of the

Este es un artículo Open Access bajo la licencia Creative Commons AtribuciónNoComercial-Compartirlgual 4.0

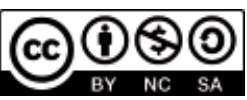


Methodological Guide for the ranking of attractions and generation of tourist spaces in Ecuador. The first stage was used with its two initial phases, and to facilitate the analysis of the criteria, a weighted percentage was applied to the evaluation criteria, in order to make statistical graphs of the attractions, which allowed us to identify that the tourism potential lies in the cultural-heritage tourism modality and that its complementary attractions, despite having many weaknesses, can be developed with proper management.

Keywords: tourism potential, planning, evaluation criteria, tourism diagnosis, sustainable development, tourism.

\section{Introducción}

El sector turismo en la actualidad avanza a grandes pasos, particularmente porque abarca múltiples áreas, e implica diversos ámbitos, por ello se considera un fenómeno social, político, cultural y económico (Bunghez, 2016). El gran volumen y la complejidad de la oferta de destinos han llevado al desarrollo de las industrias de viajes y turismo, esto se refleja en una creciente demanda de la población por una gama cada vez mayor de bienes y servicios de consumo (Sofronov, 2018), dando como resultado un aumento del flujo de la economía de la comunidad receptora donde residen estos atractivos dentro de su territorio. Como consecuencia, este aumento en el consumo turístico, causado por movimientos estacionales masivos de la población hacia ciertos destinos de interés turístico, resulta favorable al desarrollo de la economía de estos sectores que permiten el desarrollo de la actividad turística (Ivars, Rodríguez y Vera, 2013).

El turismo a menudo se ha visto como un elemento clave en el desarrollo de lugares, que han adoptado estrategias destinadas a ganar una posición competitiva y afirmar su identidad en comunicación con los turistas (Morgan, Pritchard y Pride, 2011). Sin embargo, los destinos turísticos se enfrentan a una variedad cada vez mayor de desafíos y oportunidades (Oliveira, 2014), no simplemente por atraer turistas durante las temporadas con una mayor afluencia, o porque los territorios alternativos pueden ofrecer instalaciones y posibilidades similares para visitar (Ashworth y Kavaratzis, 2010). Por el contrario, en la actualidad para desarrollar una fuerte competitividad turística debe existir una buena planificación, para así administrar correctamente los recursos con un potencial turístico notable en el territorio; para identificar estos recursos es necesario evaluar su potencialidad como punto de inicio, considerando criterios que están presentes dentro de todo producto turístico que está en desarrollo. Por ello, esta investigación se centra en las parroquias del cantón Zaruma, ubicado en la provincia de El Oro.

Zaruma, declarada Pueblo Mágico del Ecuador, oficialmente se llama Villa Real de San Antonio del Cerro de Oro de Zaruma, proviene de dos voces quechuas: sara = maíz, y uma = cabeza, al traducirse al español. Se le atribuye estas denominaciones por el maíz existente en el lugar, y por el oro de las minas, cuyo color se asemeja al de una mazorca. Se eleva sobre rocas gigantes y cerros minerales, está rodeada por cordilleras y ríos (Universidad Técnica de Machala [UTMACH], 2013). 
Es una ciudad localizada en el sur de la República del Ecuador, en la provincia de El Oro, asentada en el callejón interandino, en las estribaciones de la Cordillera de Vizcaya que se desprende de la Cordillera de Chilla. Es conocida por su arquitectura de la era republicana, sus minas de oro, tradiciones, su cultura, arte, café, su gente amable, la belleza de sus mujeres y por haber sido fundada por el español Alonso de Mercadillo. En 1998 el Instituto de Patrimonio Cultural del Ecuador inscribió a la ciudad de Zaruma como una ciudad elegible para pertenecer a la lista de Patrimonio Cultural de la Humanidad ante la UNESCO (UNL, PNUD, SNGR, 2012).

\section{Figura 1}

Mapa del cantón Zaruma

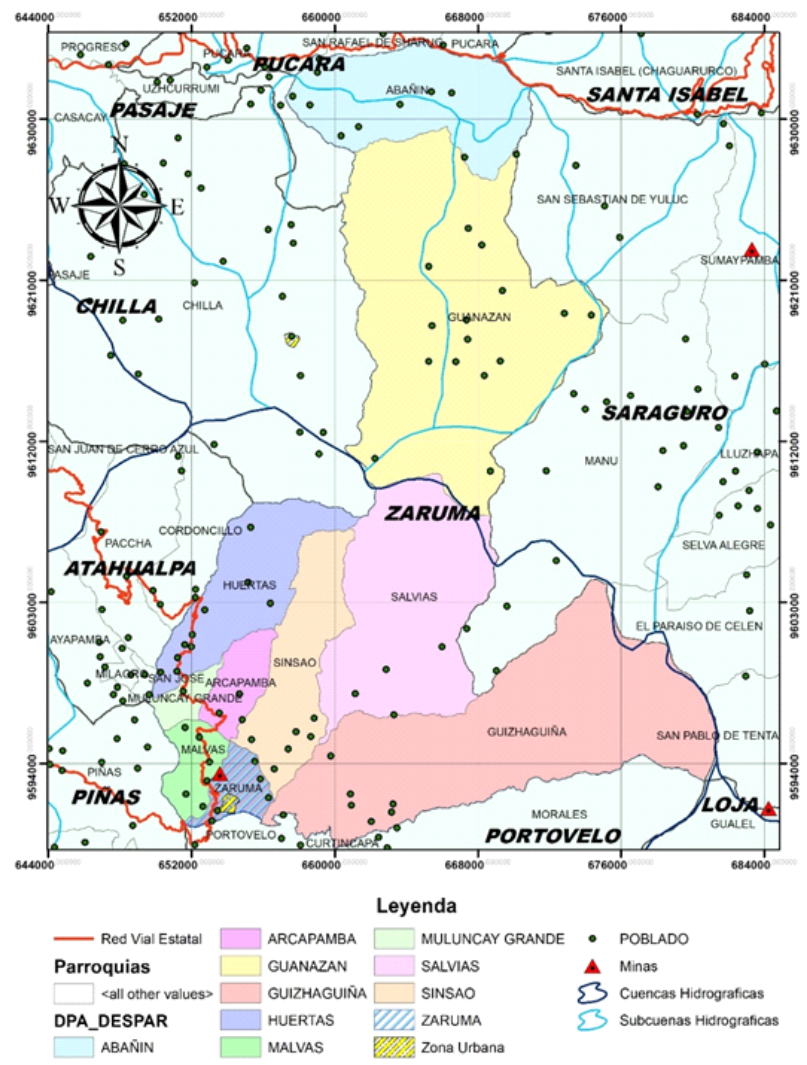

Nota. Elaboración con el software ArcMap 10.6.1

En el ámbito turístico, es uno de los principales lugares para visitar en la provincia de El Oro, su arquitectura, gastronomía y la hospitalidad de su gente, la hacen un destino a considerar para viajar con familia o amigos. El paseo por este patrimonio cultural nacional es un viaje a través de la historia y un reencuentro con la paz, que se refleja en sus atractivos turísticos los cuales agradan a todo turista que la visita. Los principales datos del cantón se presentan en la Tabla 1. 
Tabla 1

Datos generales del cantón Zaruma

\begin{tabular}{|c|c|c|}
\hline Nombre del cantón & \multicolumn{2}{|l|}{ Zaruma } \\
\hline Fecha de creación del cantón & \multicolumn{2}{|c|}{25 de junio de 1824} \\
\hline $\begin{array}{l}\text { Proyección poblacional al } \\
2019 \text { según el INEC }\end{array}$ & \multicolumn{2}{|c|}{25651 habitantes } \\
\hline Extensión & \multicolumn{2}{|c|}{6515912 ha } \\
\hline \multirow{4}{*}{ Límites } & \multicolumn{2}{|c|}{ Norte: Pucará y Santa Isabel, provincia del Azuay } \\
\hline & \multicolumn{2}{|c|}{ Sur: cantón Portovelo } \\
\hline & \multicolumn{2}{|c|}{ Este: Saraguro, provincia de Loja } \\
\hline & \multicolumn{2}{|c|}{ Oeste: Piñas, Atahualpa, Chilla y Pasaje } \\
\hline Rango altitudinal & \multicolumn{2}{|c|}{400 a 3920 m s.n.m. } \\
\hline \multirow{8}{*}{ Parroquias } & Urbana & Zaruma \\
\hline & \multirow{7}{*}{ Rurales } & Abañin \\
\hline & & Arcapamba \\
\hline & & Guanazán \\
\hline & & Güizhagüiña \\
\hline & & Huertas \\
\hline & & Malvas \\
\hline & & Muluncay Grande \\
\hline \multirow{3}{*}{ Actividades económicas } & \multicolumn{2}{|l|}{ Sinsao } \\
\hline & \multicolumn{2}{|l|}{ Salvias } \\
\hline & \multicolumn{2}{|c|}{$\begin{array}{l}\text { Agricultura, ganadería, silvicultura, pesca, explotación de minas } \\
\text { y canteras, construcción, comercio, enseñanza, entre otras. }\end{array}$} \\
\hline
\end{tabular}

Nota Información de GAD Municipal de Zaruma, 2014 (Instituto Nacional de Estadísticas y Censos [INEC], 2010).

En esta investigación nos planteamos como objetivo analizar el potencial turístico por medio de los nueve criterios de evaluación del Ministerio de Turismo del Ecuador (MINTUR), para conocer la realidad del atractivo y generar bases sólidas en una planificación sostenible a futuro, puesto que el campo de estudio carece de la misma. Para dicho fin, nos enfocamos en la primera etapa con sus dos fases. El punto de partida consistió en realizar una visita in situ, es decir en el mismo lugar o campo de estudio. Las parroquias rurales Malvas, Muluncay, Salvias, Sinsao, y su cabecera cantonal urbana la ciudad de Zaruma fueron elegidas por su accesibilidad para la realización del estudio. Durante la visita a los atractivos ubicados en las parroquias mencionadas se procedió a la recolección de datos mediante encuestas a la comunidad receptora. La herramienta metodológica se denominó Ficha para el levantamiento y jerarquización de atractivos turísticos; la información se corroboró con fuentes oficiales como el Plan de Desarrollo y Ordenamiento Territorial (PDOT) del cantón, permitiendo evaluar a los atractivos basados en los criterios determinados.

Con la información recopilada y digitalizada se obtuvo automáticamente, por medio de la matriz o ficha, el nivel jerárquico y el valor ponderado por cada criterio de evaluación de los atractivos, la suma de estos permitió conocer el nivel jerárquico. Sin embargo, este estudio se centra en la potencialidad de dichos criterios que permiten definir a un atractivo como recurso, producto o identificar su potencial turístico. 
Con estos valores se procede a la generación del Promedio de Porcentaje de Ponderación (PPP) indicador usado para realizar gráficos estadísticos e identificar mediante un análisis crítico cuáles son sus fortalezas o debilidades predominantes en el campo de estudio, con la finalidad de identificar la modalidad turística más adecuada a desarrollarse y sentar bases objetivas para un plan de desarrollo turístico sostenible por parte de la Gobernanza.

\section{Revisión de literatura}

El potencial turístico puede ser definido como la suma de posibilidades que el ambiente natural y social pone a disposición para desarrollar actividades turísticas, donde el enfoque principal se centra en la activación de la actividad para alcanzar el máximo potencial de un territorio (Mikery y Pérez, 2014). Esta potencialidad es la que permite identificar los aspectos presentes para determinar y/o implementar una modalidad turística, es decir, el tipo específico de turismo que se ejerce en dicha zona o territorio (Ibañez y Cabrera, 2011).

El conjunto de recursos que posee una comunidad o un lugar podrían transformarse en atracciones turísticas o productos terminados. Sin embargo, el potencial turístico no debe entenderse únicamente desde una perspectiva basada en los recursos, los factores operativos dentro del sistema turístico son adiciones importantes sobre el potencial turístico (Yan, Gao y Zhang, 2017). Se enfatiza en las necesidades de los sitios turísticos referidas a instalaciones, gobernanza o entes reguladores públicos, servicios, infraestructura, etc., para que las atracciones puedan estar aptas para los visitantes, y con una planificación basada en los ejes de la sostenibilidad.

La sostenibilidad es una tendencia relacionada con el desarrollo y la planificación (Niedziólka, 2012). En lo que se refiere al desarrollo está definida como la capacidad de satisfacer las necesidades de la generación actual sin comprometer la capacidad de las generaciones futuras para satisfacer sus propias necesidades (Cooper, Fletcher, Gilbert, Fyall y Wanhill, 2005) en los ejes natural, cultural y económico. Esta tendencia también es implementada en el sector turístico, que apunta a mejorar la calidad de vida de los residentes, optimizando los tres ejes mencionados y proporcionando una experiencia de alta calidad para los visitantes (Martín, Salinas, Rodríguez y Jiménez, 2017)

Un primer paso para la planificación sostenible en el ámbito turístico es el que se realiza mediante el Inventario jerarquización, y análisis de la potencialidad de un territorio. Existen muchas metodologías propuestas por organizaciones, entes o investigadores del sector turístico que se enfocan en diversas fases, para posteriormente llevar a cabo la planificación adecuada de acuerdo a las necesidades que presenta el territorio. Las primeras fases de algunas de estas metodologías se presentan en la Tabla 2. 


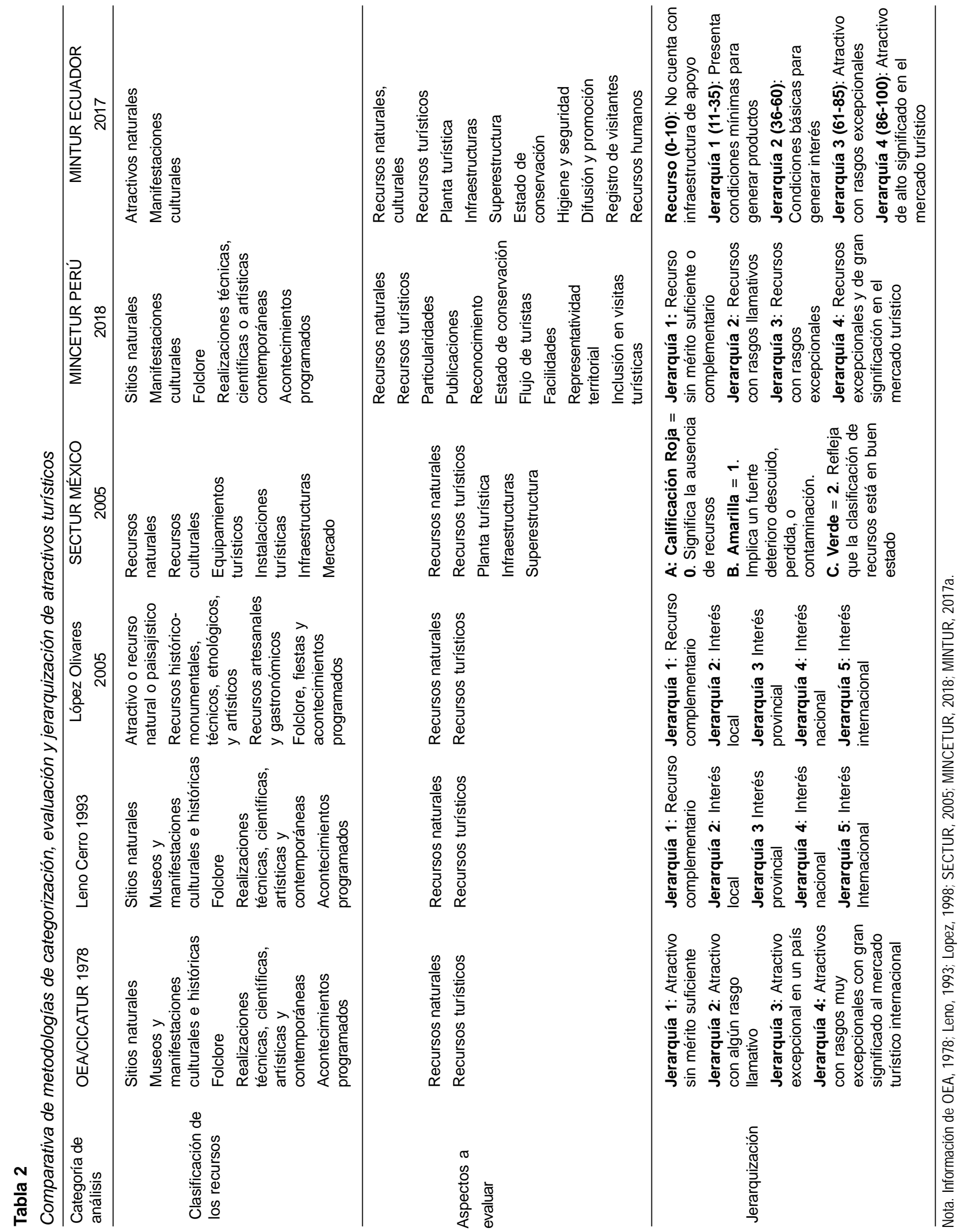


Las primeras fases de las metodologías presentan muchas similitudes en cuanto a categorías de análisis. En efecto, la aplicación de las mismas, depende del objetivo de la investigación, el enfoque, la profundidad de análisis, o el lugar de aplicación de la misma. Cada una de estas presentan sus principales ventajas, desventajas y/o limitaciones, en el sentido que unas cubren aspectos más profundos y específicos que otras. Sin embargo, cada uno de estos procedimientos tiene como finalidad la planificación a futuro del sector turístico del área de trabajo aplicada.

En este contexto se optó por la metodología del MINTUR, aplicando un enfoque territorial para esta investigación; vale decir, por la metodología aplicada según el territorio o gobernanza que la planteó, en este caso Ecuador, con la denominada Guía Metodológica para la Jerarquización de Atractivos y Generación de Espacios Turísticos del Ecuador, herramienta para identificar la potencialidad de los atractivos, por medio de lineamientos para la identificación, clasificación y valoración de los mismos, con mejores condiciones para el desarrollo (MINTUR, 2017a).

Además, la elección de esta metodología, busca el implemento de una base inicial objetiva para el futuro desarrollo de un plan de desarrollo turístico territorial sustentable, por parte de las autoridades competentes, y como este puede desarrollar al turismo del cantón Zaruma, puesto que, en la actualidad, el territorio de estudio carece de dicha planificación.

\section{Metodología}

Esta investigación responde a la línea de investigación Turismo y Sostenibilidad de la Universidad Técnica de Machala y en el grupo de investigación El Oro Turístico GRP-018, bajo el proyecto Patrimonio cultural y potencial turístico: Caso Centro Urbano de Zaruma.

Se basa en un método descriptivo con un enfoque de observación directa, realizando la visita en el lugar para conocer la realidad actual del cantón y determinar su potencial turístico. Asimismo, se empleó la guía metodológica para la jerarquización de atractivos y generación de espacios turísticos del Ecuador, la cual permite una planificación a gran escala con el fin de desarrollar el sector turístico a nivel nacional. Esta consta de dos etapas:

\section{- Etapa I}

Enfocada a la realización del Inventario de atractivos turísticos: levantamiento, registro, jerarquización y especialización de atractivos turísticos.

\section{- Etapa II}

Generación de espacios turísticos: identificación de atractivos con potencial para la generación de productos turísticos, análisis geográfico y tipificación de espacios turísticos (MINTUR, 2017a). 


\section{Figura 2}

Etapas de guía metodológica

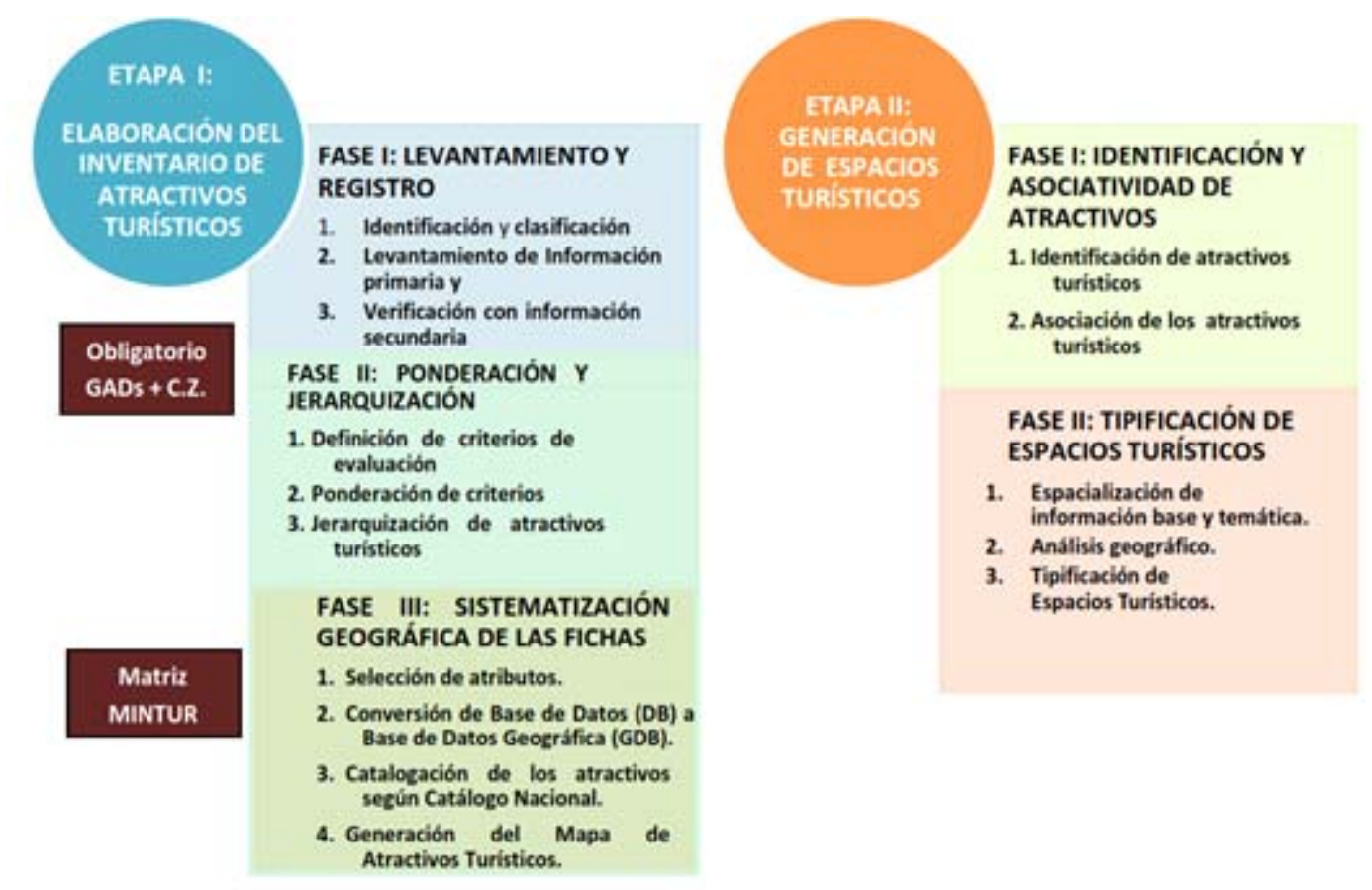

Nota Manual de atractivos turísticos (MINTUR, 2018).

Se realizó la Etapa I con sus fases de levantamiento y ponderación, las cuales permiten la evaluación del campo de estudio, conformado por los atractivos turísticos de las parroquias del cantón Zaruma.

Como instrumento metodológico para la recolección de datos se empleó la Ficha para el levantamiento y jerarquización de atractivos turísticos ${ }^{1}$ (MINTUR, 2017b), la misma que está enfocada en los nueve criterios de evaluación. Estos, permiten identificar la realidad del territorio por medio de entrevistas a la comunidad receptora y organismos de regulación, como el departamento de turismo, así como, corroborar esta información con fuentes secundarias, como el PDOT del territorio (Plan de Desarrollo y Ordenamiento Territorial). Los nueve criterios de evaluación tienen sustento en los índices de competitividad turística, publicado por el Foro Económico Mundial y en la metodología OEA.

Los criterios están clasificados según la capacidad institucional (Estado o gobiernos seccionales y el MINTUR), de incidir sobre la mejora o manejo de los destinos (MINTUR, 2018). Además, dentro de los mismos se encuentran apartados o secciones que se evalúan al momento de hacer la visita en el lugar. Se resume en la Tabla 3.

1 Matriz de la Ficha: https://servicios.turismo.gob.ec/descargas/InventarioAtractivosTuristicos/FichaAtractivos_oct2017.xls 
Tabla 3

Resumen de aspectos a evaluar en cada criterio de evaluación

\begin{tabular}{|c|c|c|}
\hline $\begin{array}{c}\text { Criterios relacionados con la } \\
\text { competitividad turística (Estado o } \\
\text { gobiernos seccionales) }\end{array}$ & Aspectos a evaluarse & $\begin{array}{l}\text { Valor de } \\
\text { ponderación }\end{array}$ \\
\hline Accesibilidad y conectividad & $\begin{array}{l}\text { Vías de acceso, ingreso a discapacitados, relación } \\
\text { distancia/tiempo, conectividad tecnológica }\end{array}$ & 18 \\
\hline Planta turística/servicios & Equipamientos y disponibilidad de servicios & 18 \\
\hline $\begin{array}{l}\text { Estado de conservación e } \\
\text { integración sitio/entorno }\end{array}$ & $\begin{array}{l}\text { Integridad y condición del sitio o entorno de los } \\
\text { atributos patrimoniales o naturales }\end{array}$ & 14 \\
\hline Higiene y seguridad turística & $\begin{array}{l}\text { Servicios básicos, control de vandalismo, limpieza e } \\
\text { iluminación del atractivo }\end{array}$ & 14 \\
\hline Políticas y regulaciones & $\begin{array}{l}\text { Consideración del atractivo dentro de la planificación } \\
\text { turística }\end{array}$ & 10 \\
\hline Actividades que se practican & Actividades que dan un valor agregado al atractivo & 9 \\
\hline \multicolumn{3}{|c|}{ Criterios relacionados con la demanda (MINTUR) } \\
\hline Difusión del atractivo & Medios de difusión y publicaciones & 7 \\
\hline Registro de visitantes y afluencia & Estimación de la demanda potencial & 5 \\
\hline Recursos humanos & $\begin{array}{l}\text { Nivel de instrucción académico y personal } \\
\text { capacitado }\end{array}$ & 5 \\
\hline & TOTAL & 100 \\
\hline
\end{tabular}

Nota. Guía metodológica para la jerarquización y generación de espacios turísticos (MINTUR, 2017a).

\section{Resultados y discusión}

Una vez recopilada la información se inició la elaboración del mapa de la ubicación de los atractivos turísticos del campo de estudio, con el software ArcMap 10.6.1. La ubicación en el mapa base es gracias a los puntos GPS recolectados por medio de la ficha, logrando delimitar visualmente los puntos estudiados. El resultado de la elaboración es reflejado en la Figura 3. 
Figura 3

Mapa de atractivos turísticos evaluados en la investigación

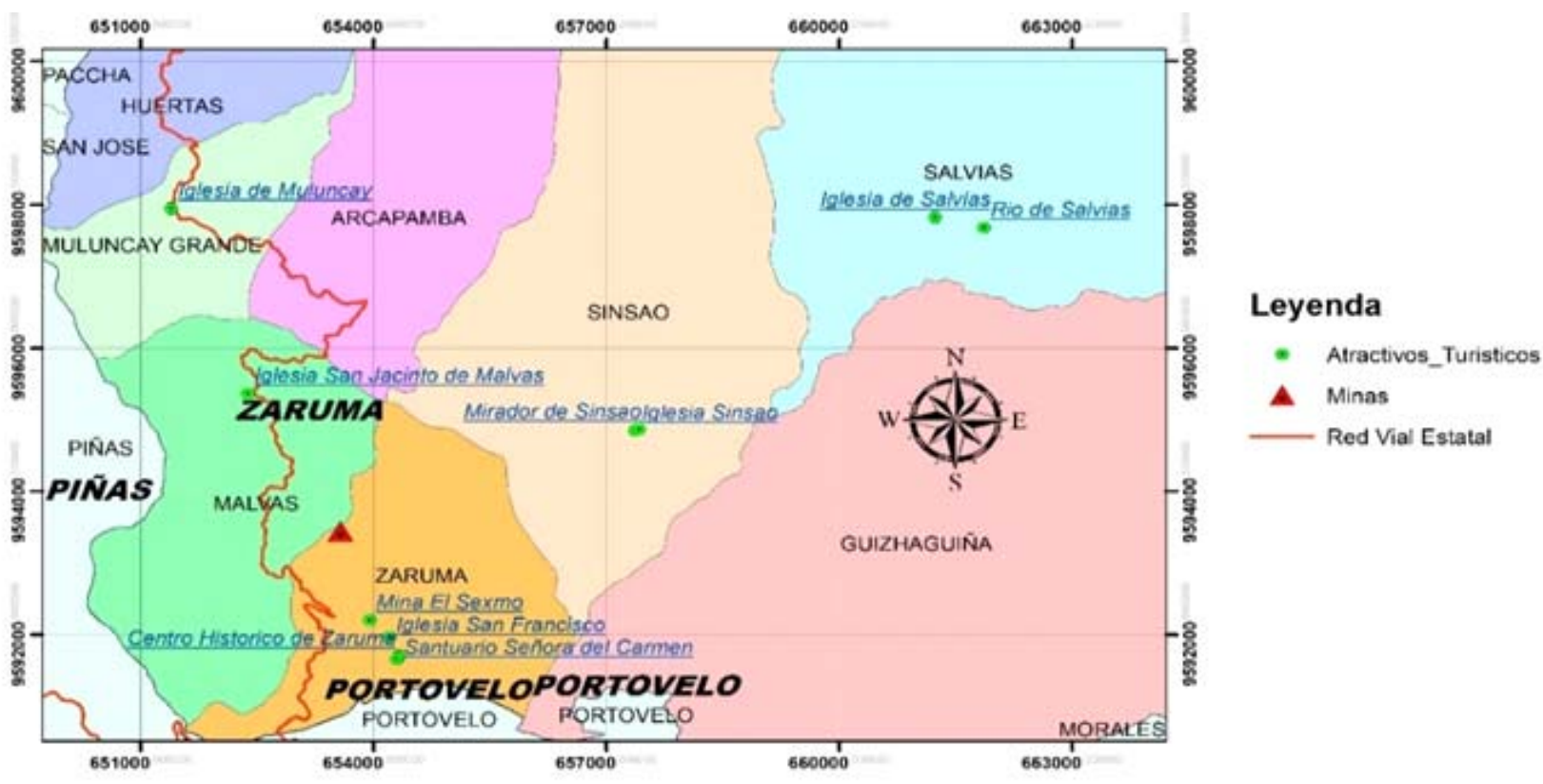

Nota. Elaboración con el software ArcMap 10.6.1

Tabla 4

Resumen de Jerarquización de atractivos

\begin{tabular}{lccccc}
\hline \multicolumn{1}{c}{ Nombre } & Categoría & Tipo & Subtipo & $\begin{array}{c}\text { Resultado de } \\
\text { evaluación } \\
\text { técnica }\end{array}$ & Jerarquía \\
\hline Centro histórico Zaruma & MC & Arquitectura & IC & 67 & 3 \\
Mina El Sexmo & MC & Arquitectura & IC & 56.9 & 2 \\
Santuario Sra. del Carmen & MC & Arquitectura & IC & 56.9 & 2 \\
Iglesia San Francisco & MC & Arquitectura & IC & 56.9 & 2 \\
Iglesia de Muluncay & MC & Arquitectura & IC & 56.1 & 2 \\
Iglesia San Jacinto & MC & Arquitectura & IC & 57.6 & 2 \\
Iglesia de Salvias & MC & Arquitectura & IC & 44.1 & 1 \\
Iglesia Sinsao & MC & Arquitectura & IC & 45.6 & 1 \\
Mirador de Sinsao & AN & Observaciones & Miradores & 45.6 & 1 \\
Río de Salvias & AN & Ríos & Río & 39.1 & 1 \\
\hline
\end{tabular}

Nota. Trabajo de campo. MC: Manifestaciones Culturales, IC: Infraestructura Cultural, AN: Atractivos Naturales 
En la Tabla 4 se presentan los valores referenciales para identificar la jerarquía que se establece en la Tabla 2 (MINTUR ECUADOR). Además, los atractivos predominantes analizados durante el estudio, son los de categoría cultural. Sin embargo, el territorio también es poseedor de belleza paisajística de índole natural. No obstante, por las vías de tercer orden, y el tiempo que toma llegar a los destinos, fueron el principal impedimento al ampliar el rango investigativo del estudio, siendo el primer aspecto limitante del mismo.

El atractivo más representativo se encuentra en la cabecera cantonal urbana del cantón Zaruma, conformado por su centro histórico. Esta zona urbana se desarrolla sin patrones preestablecidos, por las irregularidades de la topografía (GAD Municipal de Zaruma, 2014). Según el MINTUR (2017a) al poseer la jerarquía 3 define al atractivo con rasgos excepcionales, capaz de motivar por sí solo una corriente potencial de visitantes.

Los demás atractivos estudiados poseen una jerarquía menor, estando entre los rangos de jerarquía 1 y 2. A pesar de no tener un índice jerárquico mayor por sus características intrínsecas, estos atractivos complementan la experiencia de visita del territorio, al ofrecer al turista una diversificación más amplia de destinos a visitar durante su estadía.

Una vez culminada la elaboración del mapa con los atractivos estudiados, en conjunto con la categorización y jerarquización de los mismos, se procede al análisis cuantitativo de los criterios de evaluación, permitiendo evaluar objetivamente los principales aspectos que presenten condiciones para el desarrollo turístico del campo de estudio.

Los valores de los criterios de evaluación, llamados valores de ponderación, son generados automáticamente una vez digitalizados por medio de la ficha utilizada para la recolección de información, permitiendo diagnosticar el potencial turístico, a fin de sugerir acciones para su impulso o el mejoramiento de sus condiciones.

Además, se ha añadido un cálculo adicional, comprendido por el porcentaje de ponderación, indicador propuesto en la Tabla 5, con el fin de facilitar el análisis cuantitativo de gráficos estadísticos, siendo el resultado de la siguiente operación:

$$
\text { Porcentaje de Ponderacion }(\% \text { Pond })=\frac{\text { Resultado de Ponderacion del Atractivo }(R P A)}{\text { Ponderacion estándar }(M I N T U R)} \times 100
$$

Ponderación del atractivo (RPA): Valor obtenido por la evaluación in situ en el atractivo, generado por la ficha.

Ponderación estándar (MINTUR): Valores determinados para cada criterio, estandarizados por el Ministerio de turismo en Ecuador. 


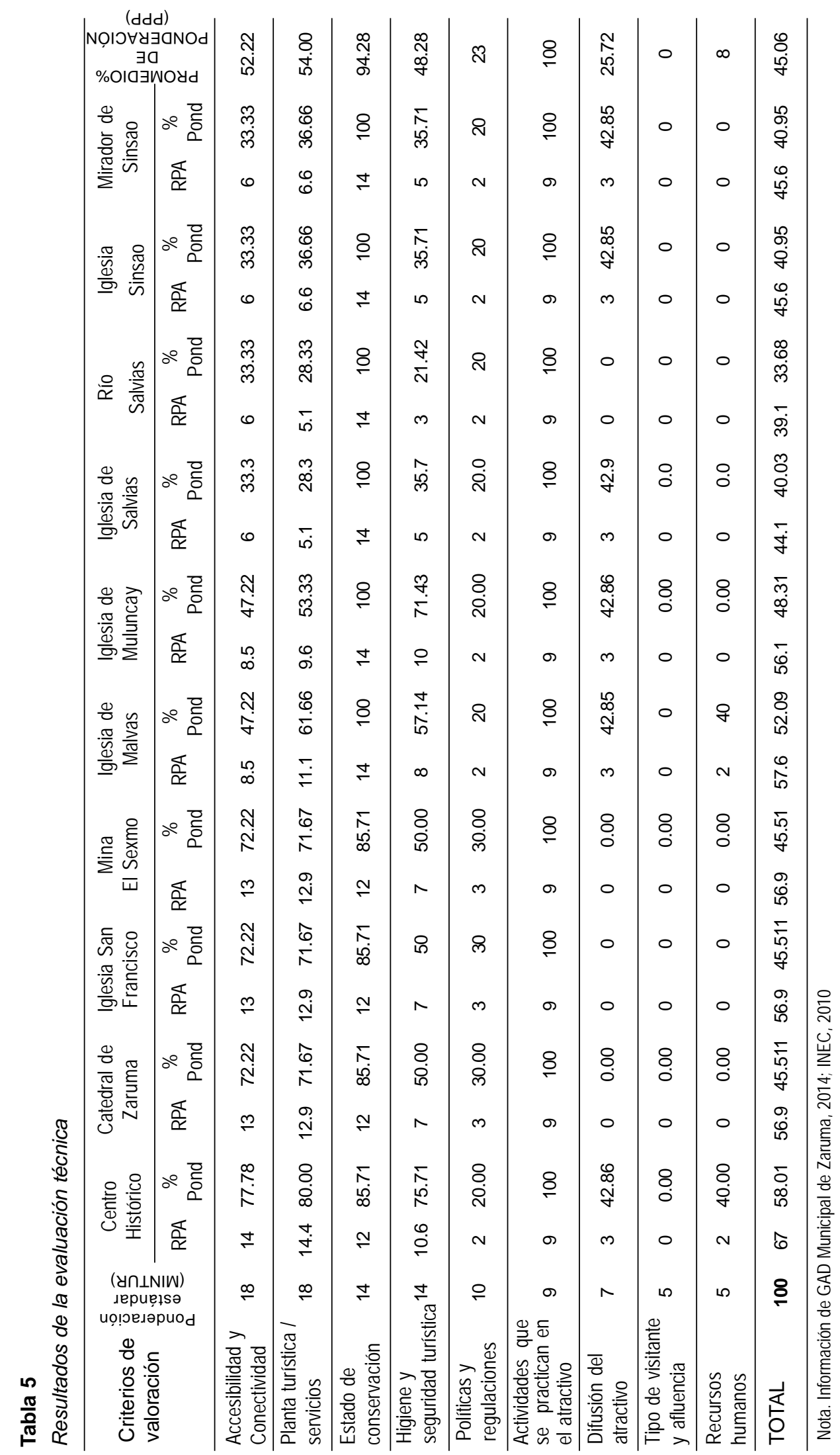


Los valores cuantitativos determinados por la evaluación técnica, están reflejados en gráficos estadísticos. Se usaron los valores de los Porcentajes de Ponderación para las Figuras 4 y 5 y el Promedio de Porcentaje de Ponderación (PPP) para la Figura 6, valores ubicados en la Tabla 5. Referente al PPP, es el promedio de los porcentajes de ponderación obtenidos de los atractivos estudiados. Con estos valores se procede a realizar el análisis de todos los destinos; según el valor obtenido de cada criterio del campo de estudio y dividiéndolos en sus respectivas categorías: culturales y naturales.

Figura 4

Potencial turístico por criterios de evaluación de atractivos culturales

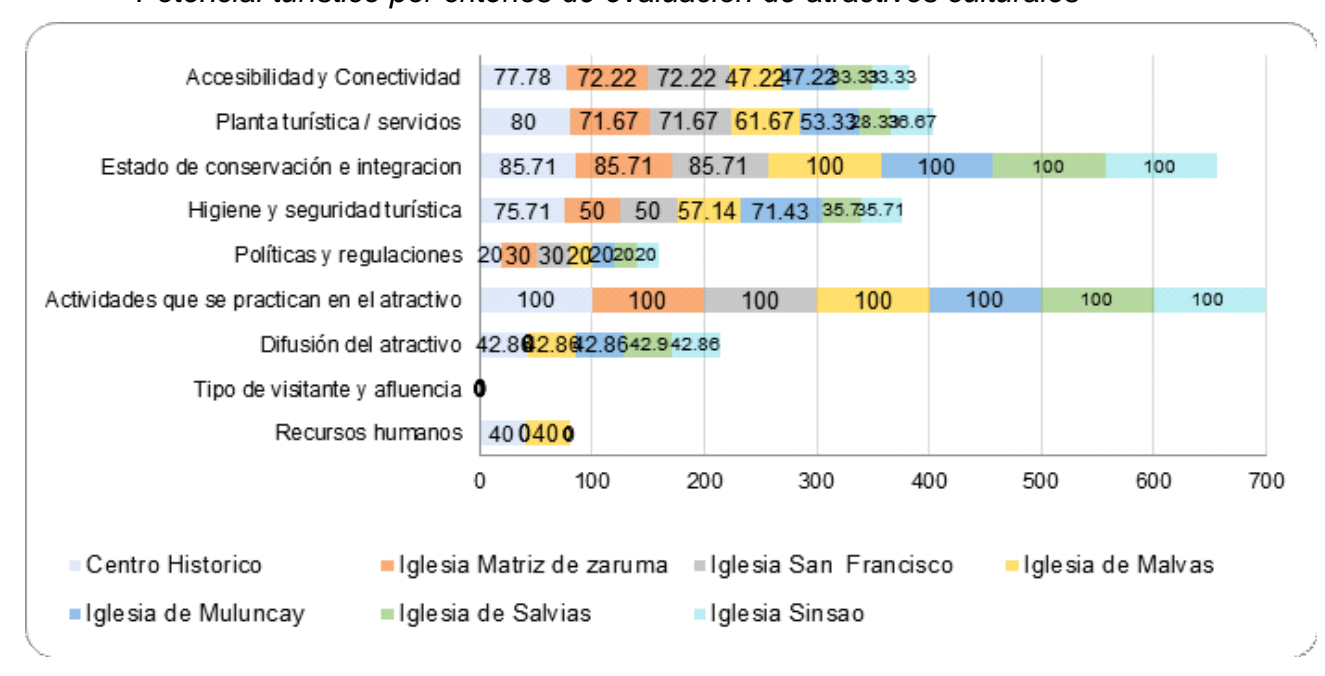

Nota Basado en los porcentajes de ponderación de atractivos culturales

\section{Figura 5}

Potencial turístico por criterios de evaluación de atractivos naturales

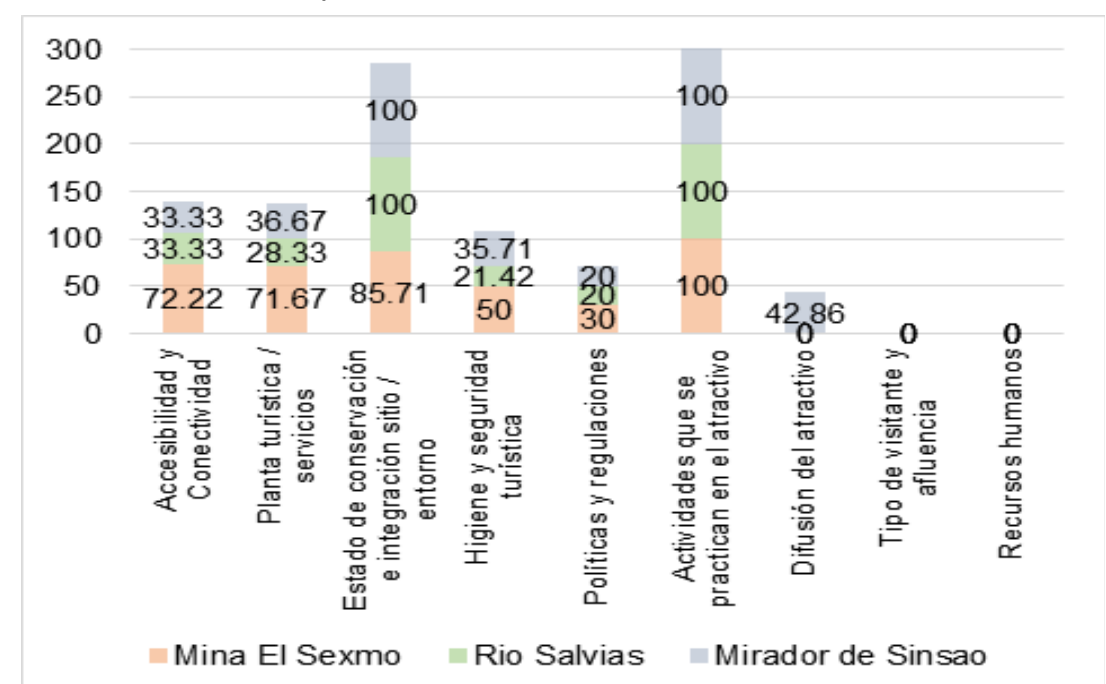

Nota. Basado en los porcentajes de ponderación de atractivos naturales 


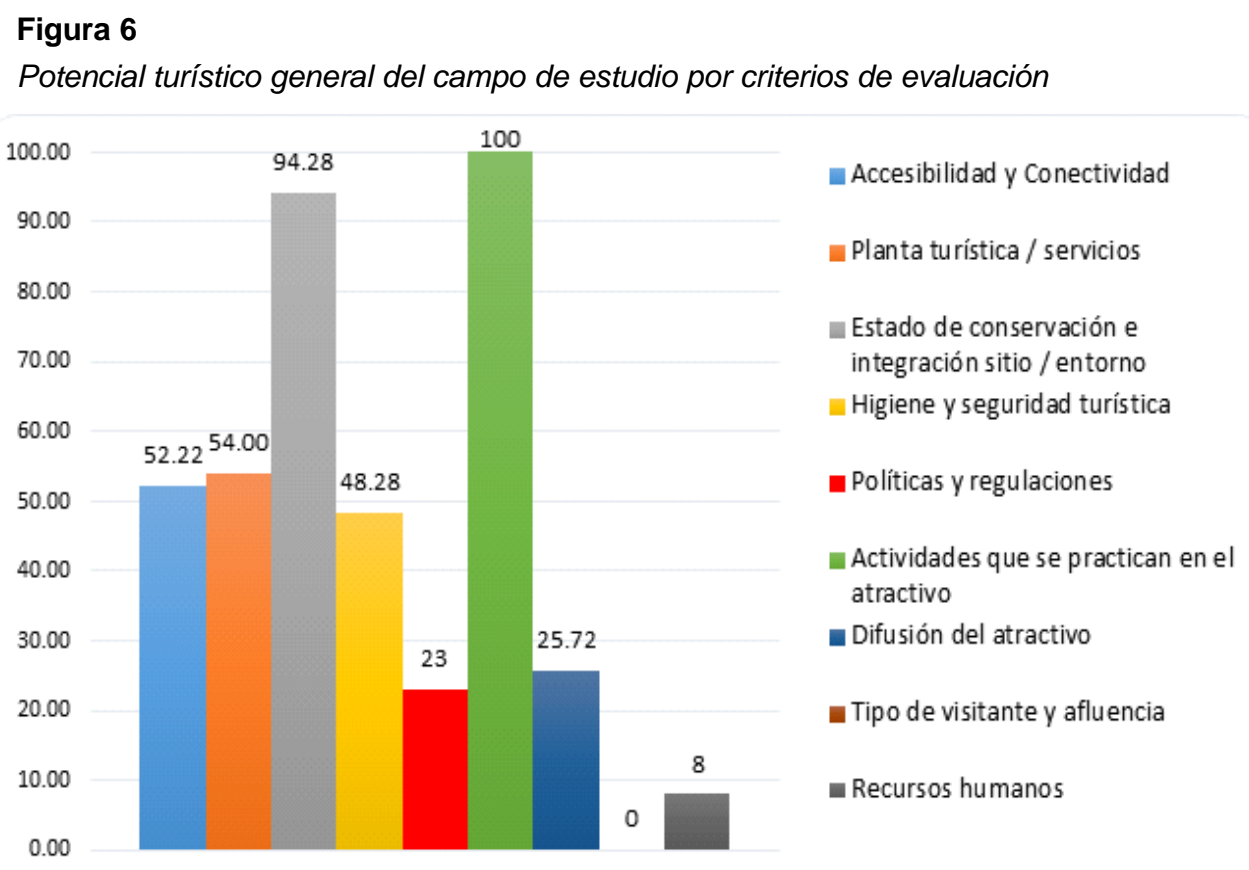

Nota. Basado en los promedios de porcentaje de ponderación (PPP)

En el análisis de la potencialidad turística se consideró a la Figura 6 como punto general de análisis, puesto que es el condensado de las Figuras 4 y 5 . Además, se tomó en cuenta un valor predeterminado de 70\%, para considerar si un criterio de evaluación representa una fortaleza; y a los porcentajes menores a este, como barreras o debilidades.

\section{Criterios relacionados con la competitividad turística (Estado o gobiernos seccionales)}

Se pudo determinar que la potencialidad turística de las principales parroquias de Zaruma, en el contexto de criterios de evaluación, está reflejada en el «Estado de conservación», y en las «Actividades que se practican en el atractivo».

Dentro del primer criterio se determina que todos los atractivos estudiados son una fortaleza predominante dentro de este contexto, debido a que estos atractivos son la principal imagen al momento de visitar las parroquias. Además, su cuidado es un factor que las autoridades y la comunidad receptora catalogan como una prioridad, no solo para fomentar el turismo, sino por considerarlos parte de su patrimonio y cultura (Barbosa, 2007).

Sobre las «Actividades que se practican en el atractivo», todos los destinos cuentan con el $100 \%$ en este criterio de ponderación, representando la mayor fortaleza del territorio. A diferencia de los demás criterios, esto demuestra la importancia del desarrollo de actividades en los atractivos turísticos, las mismas que generan rubros, producto de las motivaciones de los turistas no solo por visitar el atractivo, sino también, por realizar otras actividades fuera de lo cotidiano (Narváez y Fernández, 2010). 
No obstante, existe un mayor número de barreras o debilidades que dificultan directamente en el desarrollo turístico, debido a que de los nueve criterios de evaluación, siete son debilidades.

Los criterios de evaluación representativos mencionados anteriormente, reflejan la realidad del cantón Zaruma debido a su estructura patrimonial, la misma que debe estar en óptimo estado para recibir visitantes, siendo esta, la principal característica que incide directamente en su demanda, y cuyo posicionamiento en los mercados turísticos está dado por las tradiciones culturales de la población receptora (Walker, 2010).

Sin embargo, dentro de un territorio también existe la presencia de debilidades que impiden el desarrollo turístico, como el caso de la «Accesibilidad y la conectividad». De estos factores, dependen las posibilidades de desplazamiento de los viajeros (Muñoz y Torres, 2010), siendo un componente de la industria y un puente entre los turistas y los destinos turísticos (Wang, Niu y Qian, 2018). Este aspecto es clave en el desarrollo turístico de un territorio; pero es un punto desfavorable al constatar la realidad del campo de estudio, que se refleja en el mal estado de las vías de segundo y tercer orden que sirven para el ingreso de las parroquias rurales, lo que dificulta el traslado. Mientras que las vías de acceso dentro de la parroquia urbana se encuentran en óptimo estado.

La planta turística está representada por una serie de servicios y tareas, para satisfacer las necesidades del consumidor (Smith y Eadington, 1992). Este criterio de evaluación está catalogado como una debilidad, con un 54\% del resultado obtenido, cuyo valor es perteneciente en su mayoría a la delimitación urbana, es decir, la ciudad de Zaruma. En este sector se encuentran la mayoría de servicios turísticos; a diferencia de las parroquias rurales no cuentan con el servicio de alojamiento. Sí funcionan pequeños quioscos que ofrecen el servicio de alimentos y bebidas que, en conjunto con la belleza paisajística y cultural, deleitan a los turistas que visitan este lugar.

El ámbito de «Higiene y seguridad turística», es también considerado como una debilidad, debido a que no cuentan con señaléticas basadas en los atractivos que conforman su oferta turística, con el fin de orientar el flujo de personas hacia los mismos e informar de los servicios disponibles (Tituaña, 2013). Además, cabe mencionar que la seguridad en general, se encuentra visible mayoritariamente en la parroquia urbana por la autoridad local. Al contrario, en las zonas rurales según los pobladores, al ser sectores pequeños, la delincuencia o vandalismo no se observa por su empoderamiento en valores y cultura.

La mayor autoridad regulatoria dentro del cantón Zaruma es el GAD municipal, y sus funciones dentro del ámbito turístico se encuentran claramente descuidadas, según los resultados de la investigación en el campo «Políticas y regulaciones», por lo que es catalogada como una debilidad. Esta situación es determinada por la ausencia de un plan de desarrollo turístico territorial en todas las parroquias que conforman el estudio de campo. Cabe mencionar que cada una de ellas cuentan con su respectivo PDOT (Plan de desarrollo y ordenamiento territorial), herramienta de planeación para el desarrollo físico del territorio y la utilización del suelo (FENALCO, 2013). 
Los aspectos evaluativos que son correspondientes a las competencias del Estado o gobiernos seccionales, tienen un índice cuantitativo aceptable dentro del marco de referencia de valores ponderados establecidos, de manera estándar dentro de la metodología utilizada, a excepción del apartado de «Políticas y regulaciones». Este ámbito es una de las principales debilidades que puede presentar el desarrollo turístico dentro de un territorio, debido a la ausencia de planificación y acciones necesarias de la gobernanza, dando como resultado un lugar secundario y de menor importancia al turismo.

Cabe resaltar que esta situación no solo es recurrente en el gobierno seccional, en este caso el GAD, puesto que los criterios relacionados con la demanda (MINTUR) están en el mismo camino. La respuesta es claramente visible y reflejada en los resultados obtenidos en la Figura 6. Es decir, siendo el MINTUR principal ente regulador del turismo, tiene menos competencias a nivel país, y el GAD del territorio puede dejar en segundo plano las competencias turísticas que el MINTUR les delega a nivel nacional. Sin embargo, esto no sucede en todo el país, por ejemplo, las ciudades como Quito, Cuenca, Guayaquil, Loja, Baños, entre otras, son principales destinos turísticos por excelencia del país.

Si bien el desinterés o la no predisposición de la gobernanza en su totalidad, puede afectar directamente el turismo de un determinado territorio, no todo recae sobre la misma. Cabe destacar que en el ámbito turístico no solo está el sector público, puesto que muchas atracciones o destinos también son de propiedad privada. Este sector, al igual que la comunidad receptora y demás actores del turismo, son una parte fundamental que complementan el desarrollo de este sector, donde con un trabajo sinérgico, reciproco y planificado puede dar un resultado positivo a la gestión turística sustentable del territorio.

\section{Criterios relacionados con la demanda (MINTUR)}

A pesar de que los principales criterios de evaluación son considerados como fortalezas en algunos atractivos, esto no es igual en los últimos aspectos, como la «Difusión del atractivo», con el hecho de que ningún atractivo presenta el porcentaje mínimo requerido para ser considerado como una ventaja o fortaleza. Al contrario, estos se presentan como una debilidad, por la escasa promoción turística tanto digital como física por parte de las autoridades competentes, y por las empresas prestadoras de servicios turísticos como agencias de viajes. Por esto poseen un déficit en este criterio, cuya actividad está conformada por un conjunto de acciones e instrumentos que tienen como finalidad estimular el desarrollo del desplazamiento turístico, al igual que la mejora en la operación de esta industria en el territorio (Mendes, Biz y Gándara, 2013).

Dentro de los últimos criterios de evaluación, se encuentran el «Tipo de visitante y afluencia» y «Recursos humanos», ambos considerados como debilidades por su menor rango de porcentajes, reflejados por la inexistencia de un registro de visitantes, lo cual impide la recolección de datos y estudios de la afluencia de demanda turística. Por otro lado, el segundo criterio mencionado hace hincapié en el escaso personal capacitado en el área turística dentro de todas las parroquias estudiadas, ocasionado por un carente nivel de importancia en contratar personal especializado en turismo o de capacitarlo en esta rama, lo que impide el aprendizaje de nuevos métodos, técnicas, e ideas que orienten a la mejora continua de la gestión del establecimiento (Parra y Mendieta, 2016). 
En comparación con los criterios de competitividad turística, los criterios relacionados con la demanda cuentan con un índice de evaluación significativamente menor en todos los aspectos evaluados, sobre todo en atractivos naturales, puesto que no cuentan con las facilidades necesarias como para ser un producto turístico. Esta situación comprende, no solamente al campo de estudio, sino también los atractivos ubicados en las demás parroquias remotas del cantón, y que no formaron parte de esta investigación.

Por lo general, ambos criterios de competitividad turística y de demanda comparten ciertas similitudes en las razones de sus valores de ponderación explicadas anteriormente, tanto en los atractivos culturales como naturales, pero es notable que existe un mejor control o gestión por parte de la gobernanza en los atractivos culturales urbanos, los mismos que conforman la principal imagen del cantón.

\section{Conclusiones}

Dentro del campo de estudio turístico, el análisis crítico y la jerarquización de atractivos basados en su potencialidad, es uno de los pilares a ejecutarse para conseguir un correcto desarrollo sostenible y desarrollar un producto turístico con potencial para atraer visitantes. Específicamente, dentro del campo de estudio analizado, muchos atractivos cuentan con fortalezas resaltantes, que con una gestión adecuada pueden desarrollarse efectivamente, aprovechando los recursos de manera correcta, y el cantón Zaruma es un territorio con una potencialidad intrínseca neta de tipo cultural-patrimonial, en el que se desarrolla esta modalidad turística basada en este último aspecto.

Muchos de los atractivos estudiados cuentan con debilidades más que con fortalezas, esta situación aunque es reparable con una gestión y desarrollo óptimo de los atractivos, sigue siendo una realidad presente dentro de los territorios, ya sean nacionales o internacionales. Añadiendo que el potencial de sus atractivos no ha sido desarrollado de una manera adecuada, o pertinente, por sus autoridades o actores respectivos.

Cabe destacar que las debilidades o barreras que más resaltan se presentan dentro del territorio y los atractivos analizados son competencias principales de los entes públicos, cuyas competencias están descuidadas con respecto a la actividad turística, como la accesibilidad, políticas y regulaciones, y difusión del atractivo.

Sin embargo, no solo basta con señalar las debilidades con las que cuenta el territorio, muchas de las fortalezas son las que han posicionado actualmente al cantón Zaruma como uno de los principales destinos para visitar dentro de la provincia de El Oro, que se destaca por su belleza paisajística, actividades que se realizan y su estado de conservación patrimonial y empoderamiento de la comunidad. Todo este conjunto de factores o fortalezas desarrolladas ocurren por el accionar de los principales actores involucrados en el sector turístico, aunque presenta falencias en varios aspectos señalados anteriormente referentes a su aplicación, ya sea por falta de presupuesto, motivación o desconocimiento del personal competente en el área.

Por lo tanto, es necesario establecer un plan de acción, tomando como base el presente estudio, cuyas limitaciones se encuentran principalmente en el rango investigativo del mismo, o el cumplimiento completo de todas las etapas de la metodología utilizada. Sin embargo, es el cimiento para el planteamiento de objetivos que conlleven a una planificación turística sustentable, que cuente con lineamientos basados en el correcto manejo y 
aplicación de las directrices competentes en la gestión turística del territorio, agregando un valor añadido y un mayor desarrollo.

\section{Referencias}

Ashworth, G. y Kavaratzis, M. (2010). Towards effective place brand management: branding european cities and regions. Journal of Regional Science, 649-650. doi:10.1111/j.1467-9787.2011.00737.x

Barbosa, M. (2007). La política del turismo cultural y el diseño de producto turístico para el turismo cultural. Caso: corredor turístico Bogotá-Boyacá-Santander. EAN Escuela de Administracion de Negocios, 105-122.

Bunghez, C. (2016). The Importance of Tourism to a Destination's Economy. Journal of Eastern Europe Research in Business \& Economics, 1-9. doi:10.5171/2016.143495

Cooper, C., Fletcher, J., Gilbert, D., Fyall, A. y Wanhill, S. (2005). Tourism: Principles and Practice. Norway: Pearson.

Federación Nacional de Comerciantes (FENALCO). (2013). ¿Qué es un plan de ordenamiento territorial y para qué sirve? https:// www.fenalcobolivar.com/desarrollo-sectorial/que-es-un-plan-de-ordenamiento-territorial-y-para-que-sirve-1487

GAD Municipal de Zaruma. (2014, noviembre). GAD Municipal de Zaruma. http://app.sni.gob.ec/sni-link/sni/PORTAL_SNI/ data_sigad_plus/sigadplusdiagnostico/PDOT_Zarumadiagnostico5nov2014_15-11-2014.pdf

Ibañez, R. y Cabrera, C. (2011). Teoría general del turismo: un enfoque global y nacional (vol. 1). México: Serie Didáctica.

Instituto Nacional de Estadística y Censo (INEC). (2010). Proyección de la población ecuatoriana, por años calendario, según cantones 2010-2020. https://www.ecuadorencifras.gob.ec/documentos/web-inec/Poblacion_y_Demografia/Proyecciones_ Poblacionales/proyeccion_cantonal_total_2010-2020.xlsx

Ivars, J., Rodríguez , I. y Vera, J. (2013). The evolution of mass tourism destinations: New approaches beyond deterministic models in Benidorm (Spain). Tourism Management, 34, 184-195. http://doi:10.1016/j.tourman.2012.04.009

Leno, C. (1993). Técnicas de evaluación del potencial turístico. Madrid: Ministerio de Industria, Comercio y Turismo.

Lopez, D. (1998). Técnicas de evaluación del potencial turístico. Ministerio de Industria, Comercio y Turismo. Castellon: Técnicas de evaluación del potencial turístico. Ministerio de Industria, Comercio y Turismo.

Martín, J., Salinas, J., Rodríguez, J. y Jiménez, J. (2017). Assessment of the Tourism’s Potential as a Sustainable Development Instrument in Terms of Annual Stability: Application to Spanish Rural Destinations in Process of Consolidation. Sustainability, 9(1692), 1-20. doi:10.3390/su9101692

Mendes, G., Biz, A. y Gándara, J. (2013). Innovación en la promoción turística en medios y redes sociales. Estudios y Perspectivas en Turismo, XXII(1), 103-119. https://dialnet.unirioja.es/servlet/articulo?codigo=5131415

Mikery, J. y Pérez, A. (2014). Métodos para el análisis del potencial turístico del territorio rural. Revista Mexicana de Ciencias Agrícolas, 1729-1740.

Ministerio de Comercio Exterior y Turismo (MINCETUR). (2018, diciembre). Manual para la elaboración y actualización del inventario de recursos turísticos. https://www.mincetur.gob.pe/wp-content/uploads/documentos/turismo/consultorias/directoriosManuales/ Manual_para_la_Elaboaracion_y_actualizacion_del_inventario_de_recursos_turisticos.pdf

Ministerio de Turismo del Ecuador (MINTUR). (2017a). Guía metodológica para la jerarquización de atractivos y generación de espacios turísticos del Ecuador. https://servicios.turismo.gob.ec/descargas/InventarioAtractivosTuristicos/Parte1_GuiaMetodologica InventarioGeneracionEspacioTuristico2017_2daEd.pdf

Ministerio de Turismo del Ecuador (MINTUR). (2017b). Ficha para el levantamiento y jerarquización de atractivos turísticos. https:/ /servicios.turismo.gob.ec/descargas/InventarioAtractivosTuristicos/FichaAtractivos_oct2017.xls

Ministerio de Turismo del Ecuador (MINTUR). (2018). Manual de Atractivos Turísticos. https://servicios.turismo.gob.ec/descargas/ InventarioAtractivosTuristicos/MANUAL-ATRACTIVOS-TURISTICOS.pdf

Morgan, N., Pritchard, A. y Pride, R. (2011). Destination brands: managing place reputation. Place Branding and Public Diplomacy, 7(4), 316-320. doi:10.1057/pb.2011.25 
Muñoz, M. y Torres, R. (2010). Conectividad, apertura territorial y formación de un destino turístico de naturaleza. El caso de Aysén. Estudios y Perspectivas en Turismo, 19(4), 447-470.

Narváez, M. y Fernández, G. (2010). El turismo desde la perspectiva de la demanda. Lugar de estudio: Península de Paraguana - Venezuela. Scielo, 175-183. http://www.scielo.org.co/pdf/rudca/v13n2/v13n2a20.pdf

Niedziólka, I. (2012). Sustainable Tourism Development. Regional Formation and Development Studies, 3(8), 157-167.

Oliveira, E. (2014). The tourism potential of Northern Portugal and its relevance for a regional branding strategy. Advances in Hospitality and Tourism Research, 2(2), 54-78.

Organización de Estados Americanos (OEA). (1978). Metodología de inventario turístico en Quinto. Curso Interamericano de Planificación del Desarrollo Turístico. México.

Parra, S. y Mendieta, J. (2016). Capacitacion a servidores turisticos y gestion de politica publica: caso nabon. Repositorio Institucional de la Universidad del Azuay. http://dspace.uazuay.edu.ec/bitstream/datos/5528/1/11857.pdf

SECTUR. (2005). Identificación de potencialidades turísticas en regiones y municipios. México. Serie de documentos técnicos en competitividad, 8. https://gestiondedestinos.files.wordpress.com/2011/11/fasciculo81.pdf

Smith, V. y Eadington, W. (1992). Tourism alternatives: Potentials and problems in the development of tourism. Pennsylvania: University of Pennsylvania Press.

Sofronov, B. (2018). The Development of the Travel and Tourism. Annals of Spiru Haret University. Economic Series, 18(4), 123137. doi:10.26458/1848

Tituaña, S. (2013). La importancia de una correcta señalética en los lugares turísticos de la parroquia de San Jose de minas. Repositorio digital del Instituto Tecnológico Superior Cordillera. http://www.dspace.cordillera.edu.ec/bitstream/123456789/1204/1/58TURISM-13-13-1720815925.pdf

UNL, PNUD, SNGR. (2012, diciembre). Identificación del Territorio del Cantón. Repositorio Institucional del Consorcio Ecuatoriano para el Desarrollo de Internet Avanzado. http://repositorio.cedia.org.ec/bitstream/123456789/840/1/Perfil\%20territorial\%20\% 20ZARUMA.pdf

Universidad Técnica de Machala (UTMACH). (2013). El Oro. Guía turística. Machala: UTMACH. https://issuu.com/eloroturistico/ docs/revistaturisticaeloro

Walker, M. (2010). Cities as creative spaces for cultural tourism: a plea for the consideration of history. PASOS. Revista de Turismo $y$ Patrimonio Cultural, 8(3), 17-26. doi:10.25145/j.pasos.2010.08.031

Wang, D., Niu, Y. y Qian, J. (2018). Evolution and optimization of China’s urban tourism spatial structure: A high speed rail perspective. Tourism Management, 64, 218-232. doi:10.1016/j.tourman.2017.08.010

Yan, L., Gao, B. y Zhang, M. (2017). A mathematical model for tourism potential assessment. Tourism Management, 63, 355-365. doi:10.1016/j.tourman.2017.07.003

Luis Miguel Sánchez Cuenca

Egresado de la Universidad Técnica de Machala, con conocimiento en Planificación y Gestión Turística.

ORCID: http://orcid.org/0000-0002-1418-9444

Abraham Isaías Parra Prado

Egresado de la Universidad Técnica de Machala con conocimientos en Guianza Turística.

ORCID: http://orcid.org/0000-0001-8376-576X

Melissa Paulina Calle Iñiguez

Phd en Ciencias Administrativas, magister en Planificación Turística. Docente investigador de la Universidad Técnica de Machala.

ORCID: http://orcid.org/0000-0003-1615-0559 